\title{
Promoting universal financial protection: contracting faith-based health facilities to expand access - lessons learned from Malawi
}

Maureen L Chirwa ${ }^{1 *}$, Isabel Kazanga ${ }^{2}$, Giulia Faedo ${ }^{3}$ and Stephen Thomas ${ }^{3}$

\begin{abstract}
Background: Public-private collaborations are increasingly being utilized to universalize health care. In Malawi, the Ministry of Health contracts selected health facilities owned by the main faith-based provider, the Christian Health Association of Malawi (CHAM), to deliver care at no fee to the most vulnerable and underserved populations in the country through Service Level Agreements (SLAs). This study examined the features of SLAs and their effectiveness in expanding universal coverage. The study involved a policy analysis focusing on key stakeholders around SLAs as well as a case study approach to analyse how design and implementation of SLAs affect efficiency, equity and sustainability of services delivered by SLAs.
\end{abstract}

Methods: The study employed both qualitative and quantitative research methods to address the research questions and was conducted in five CHAM health facilities: Mulanje Mission, Holy Family, and Mtengowanthenga Hospitals, and Mabiri and Nkope Health Centres. National and district level decision makers were interviewed while providers and clients associated with the health facilities were surveyed on their experiences. A total of 155 clients from an expected 175 were recruited in the study.

Results: The study findings revealed key aspects of how SLAs were operating, the extent to which their objectives were being attained and why. In general, the findings demonstrated that SLAs had the potential to improve health and universal health care coverage, particularly for the vulnerable and underserved populations. However, the findings show that the performance of SLAs in Malawi were affected by various factors including lack of clear guidelines, non-revised prices, late payment of bills, lack of transparency, poor communication, inadequate human and material resources, and lack of systems to monitor performance of SLAs, amongst others.

Conclusions: There was strong consensus and shared interest between the government and CHAM regarding SLAs. It was clear that free services provided by SLAs had a great impact on the impoverished locals that used the facilities. However, lack of supporting systems, inadequate infrastructure and shortage of health care providers affected SLA performance. The paper provides recommendations to policy makers for the replication and strengthening of SLA implementation in the roll-out of universalization policy.

Keywords: Health policy, Financial risk protection, Policy analysis, Universal coverage

\footnotetext{
* Correspondence: maureenlchirwa@gmail.com

${ }^{1}$ Community Health Department, College of Medicine, Chichiri, Blantyre 3,

Malawi

Full list of author information is available at the end of the article
} 


\section{Background}

Since 2004, the Ministry of Health $(\mathrm{MoH})$ in Malawi has subsidized health facilities owned by the main faithbased provider, the Christian Health Association of Malawi (CHAM), to deliver care at no fee to the most vulnerable and underserved populations in the form of Service Level Agreements (SLAs). SLAs are a national policy priority, implemented (in the context of decentralization) at the district level, aiming at ensuring universal financial risk protection with a primary focus on maternal and neonatal interventions. This study examined the effectiveness of the SLA contracting arrangements and the reasons for this in terms of SLA design and the relationship between the government and CHAM in Malawi.

The basic health indicators in Malawi are amongst the worst globally. For instance, HIV/AIDS prevalence stands at $11 \%$ amongst adults, maternal mortality ratio is $675 / 100,000$ and under-five mortality is $112 / 1,000$ [1]. In order to address these and other health challenges, the Government of Malawi together with development partners have been implementing reforms aimed at improving efficiency and equity in the delivery of health care. To reduce the high burden of disease in Malawi, the $\mathrm{MoH}$ and its collaborating partners developed a six year Joint Program of Work 2004-2010 as a strategic means to deliver the Essential Health Package (EHP) and other interventions aimed at improving the health care delivery system [2]. The EHP is a combination of high impact and cost-effective health care interventions. The Malawi EHP has thirteen components and it focuses on the major causes of morbidity and mortality, particularly those affecting vulnerable populations. The Joint Program of Work was succeeded by the Health Sector Strategic Plan (2011-2016) whose overall goal is to improve the quality of life of all the people of Malawi [2]. The EHP is delivered free of charge to all Malawians by the public health sector. CHAM health facilities also deliver selected EHP services free to women and children under five. CHAM is an umbrella organization of most Christian-church owned health facilities; it is a very large player in the national health sector and oversees approximately $35 \%$ of all health centres in Malawi.

The 2011 Malawi Demographic and Health Survey showed that maternal mortality, as well as infant and child mortality, decreased only modestly since the last survey in 2004. There are many persistent challenges in the health sector, such as inadequate financing, shortage of drugs, health planning, drug procurement, and a lack of monitoring and evaluation [2,3]. Although $\mathrm{MoH}$ finances the purchase of some drugs and all local staffing costs in CHAM facilities, CHAM charges fees to cover other drugs and operating expenses. Further,
CHAM facilities tend to be located in the very poorest areas of Malawi, and thus their fees policy represents a financial barrier to access to those living in their catchment areas; such fees have reduced access to care and impoverished households [4]. Consequently, $\mathrm{MoH}$ encouraged District Health Offices (DHOs) to establish SLAs for the removal of user fees in CHAM. By 2011, 75 out of approximately 172 CHAM facilities were covered by SLAs and the initial SLAs had been in operation for more than 5 years.

Public health care financing in Malawi comes largely from government taxes as well as from contributions by donors to the health budget via direct budgetary support to the Ministry of Finance or through the health sector wide approach. Private financing is the second major source of health financing in Malawi. A good proportion of the population is not covered by a public facility and such communities have to make an out-of-pocket payment for use of health services at the for-profit health facilities. Private health insurance is not well developed, largely due to state provision and financing of health services, and to high levels of poverty.

The use of contracting with non-state providers has been highlighted in the literature as potentially very effective in improving the quality and coverage of care, but where further research is required $[5,6]$. Contracting has been associated with increased technical efficiency through making health facilities claimants on revenues or exposing them to competitive pressures [6]. Such reforms may also have pragmatic advantages - including the ability to bypass overly bureaucratic government procedures and overcome absorptive capacity constraints in the scale up of services [5]. Nevertheless, contracts are prone to problems associated with agency and transaction costs. Where the objectives of the contracting parties do not align there are problems with guaranteeing that the agent works in the interests of the principal as well as issues with transparency, causing 'information asymmetry'; these conflicting objectives will lead the agent to behave as a 'moral hazard', indulging in actions for its own benefit and not those of the principal. The principal is thus forced to incur costs either in relation to monitoring the agent's actions or in terms of formulating a contract to produce sufficient incentives for 'good' agent behaviour [7]. In addition, the resources consumed to develop, evaluate and renegotiate contracts themselves may be significant [5]. Such factors may well undermine the efficiency of contracting. Further, the dynamic of repeated contracts allows parties to become familiarised and to develop a relationship and a reputation, and thus adjust their actions [8]; this may enhance the contracting relationship but it may also reveal true intentions and actions and cause difficulties in the contracting process. Hence, while there may be 
gains from contracting for universal care there are also substantial risks.

The Taskforce for Health Systems Research identified non-state sector research as a priority research topic in its 2005 report. The Alliance for Health Policy and Systems Research has also made the 'Role of the NonState Sector in Health Care Provision' one of its three priority themes [9]. In particular, there is a need for improved knowledge in a number of areas, including the dynamics of the purchaser and provider relationship [6]; systems effects and equity implications [9]; the capacity of resource-constrained governments to fulfil the purchaser role; and how relationships with providers evolve over time [6]. The Alliance for Health Policy and Systems Research call for proposals was on "assessing efforts towards universal financial risk protection in low- and middle-income countries". This study assesses the efforts made by the Government of Malawi focusing on case studies.

The objectives of the study are to examine the purchaser-provider relation dynamics, where the purchaser is the government and the provider is non-state, assessing the effects of this relationship on care provision and access on the part of the beneficiary. In particular, this study aims to examine the effectiveness of SLAs, their contractual arrangements in terms of design of the agreements, and the relationship between the government and CHAM in Malawi. The specific objectives of this study are to: i) understand the governance structures, sectoral context and inter-sectoral dynamics, within which SLAs have emerged as a policy reform and to identify any obstacles to their adoption; and ii) evaluate the efficiency, effectiveness, equity and sustainability of SLAs aiming to improve the national roll-out policy, and in particular to assess how provider payment mechanisms within SLAs have impacted on coverage and user satisfaction.

\section{Research questions}

The study was guided by the following research questions: What are the factors that have constrained or supported contracting out of government services to CHAM facilities in Malawi? What determined the choice of provider payment mechanisms in SLA development? How has the design of the SLA, and in particular the choice of provider payment mechanism, affected the efficiency and equity of purchasing services, the financial accessibility of households and the sustainability of the reform? What are the lessons in terms of designing and managing contracting out of services to faith-based organizations to further universal coverage?

\section{Methods}

This study employed a mixed methods approach, whereby both quantitative and qualitative data were used. It consisted of two strands. The first was a policy analysis focusing on the key stakeholders around SLAs, their views, communication and interactions. The second component involved case studies to analyse how SLA design and implementation affected their efficiency, equity and sustainability.

\section{Sources of data}

The study recruited its participants from national, district and local levels: policy and decision makers who were involved at the establishment of SLAs, DHO and CHAM Health Facility Management teams, health care providers and clients. The study aimed to interview a maximum of 20 participants ( 10 at central, and 2 at each case study facility). A total of 155 clients from the expected 175 who visited the health care facilities for maternal and child health were recruited in exit interviews. Nine focus group discussions (FGDs) were conducted. The FGDs comprised available community members surrounding each participating facility. Quantitative data were captured from the Health Management Information System (HMIS). Data collectors collected the data using a checklist.

\section{Policy analysis}

Key documents and communications in the form of minutes and memos were reviewed and analysed to understand the context and reform development within which SLAs emerged. The relationship between government and CHAM was investigated using semi-structured interviews, policy analysis tools and techniques around both the design and implementation of the agreement [10].

\section{Questionnaire and semi-structured interviews}

The questionnaire administered to health care providers used a mixture of Likert scales and closed and open-ended questions. Quantitative data from the questionnaires was analysed using excel. Data from questions and interviews were analysed manually using a thematic content analysis. A coding tree, which was agreed upon by the research team, was developed before data collection. Following data collection and transcription, coding was done by one member of the study team. The coded data was grouped into categories and themes. After coding, the data was independently checked by another member of the study team to ensure validity.

Semi-structured interviews with key personnel both at $\mathrm{MoH}$ and $\mathrm{CHAM}$ central and district levels, were conducted in English. Participants were purposefully sampled through the National Steering Committee on SLAs. The Committee was specifically established to guide the study and was composed of members from the $\mathrm{MoH}, \mathrm{CHAM}$ and the College of Medicine who were selected according to their involvement and knowledge 
of the SLA and CHAM. Both district government officers and CHAM facility managers were interviewed to explore SLA incentives and behaviour encouragement activities. The frontline interviews were designed to supplement information collected from HMIS, annual reports and client exit interviews. The findings corroborated the documents reviewed.

\section{Exit interviews}

The client exit interviews were administered to clients who were randomly recruited after they had received health care services at each case study facility. Data on the care process were collected with attention to cost barriers, non-cost barriers and perception of clients on the service provided. The study aimed to interview a maximum of 35 clients per facility. However, due to low number of clients at some facilities, the number of people interviewed was lower. A total of 155 clients, from an expected 175, who visited the health care facilities for maternal and child health were recruited in the interview. The interviews were conducted using an interview guide and administered in the local language of Chichewa.

\section{Focus group discussions (FGDs)}

FGDs were conducted with community members nearest to the participating facilities. The FGDs comprised of women only because men felt women were more suitable given that they were the ones who accessed the services. The number of participants per group varied from five to eight. Data from FGDs complemented that from exit interviews, providing information on access and perceived effectiveness. Data from FGDs were also analysed manually using a thematic content analysis following the same process as that of the semi-structured interviews.

\section{Health Management Information System (HMIS)}

HMIS data was collected at the facility level, before and after the removal of fees and the introduction of SLAs. The aim was to solicit data on patterns of utilization and was used to assess the interventions covered by SLA at each facility. Due to the limited capacity of staff, it was not possible to access the HMIS data in some facilities. Information from HMIS was collated with the corresponding invoices to compare the cost of provision being passed on to the DHO for reimbursement.

\section{SLA case studies}

Case studies are a useful approach when complex phenomena are under study and the richness of material is more important to understand than breadth of scope [11]. Upon discussion with the National Steering Committee, five case studies in five different health facilities were recommended, leading to the modification of the criteria and selection of the facilities in liaison with CHAM. The facilities selected were:

Mtengowanthenga Hospital, Dowa District (Central region). It was the first institution to sign an SLA in 2004 covering maternal health but the contract was later revised to include child health. It caters for a catchment area population of 56,000.

Mulanje Mission Hospital, Mulanje District (Southern region). Located $10 \mathrm{~km}$ from Mulanje District Hospital. Hence, the catchment areas of the two hospitals overlap. The hospital signed an SLA in 2005, which covers maternal and child health services.

Holy Family Hospital, Phalombe District (Southern region). It signed the SLA in 2006 and is located in a rural area. The Holy Family SLA is distinct in that the hospital acts as a district hospital and is therefore used as a referral facility. It covers all elements of the EHP unlike other SLAs that cover only one or two elements.

Mabiri Health Centre, Mzimba District (Northern region). It is located in a very remote area where there is no government facility nearby. It signed an SLA in 2006 and covers both maternal and child health services.

Nkope Health Centre, Mangochi District (Southern region). The SLA in Nkope was signed in 2006 being one of the newest and covers maternal and child health.

The key characteristics for each case study choice relate to reviewing the length of the contractual relationship between the DHOs and CHAM facilities and how the presence of competition might affect this interaction.

\section{Results}

Governance structures, sectoral context and inter-sectoral dynamics within which SLAs emerged

The results of the semi-structured interviews with central and facility level managers revealed the absence of explicit and documented policies to guide the implementation of SLAs. Through these interviews, a policy analysis was performed to evaluate the relationship between government and CHAM, and how this affected SLAs' design and implementation. In relation to governance, the interviews revealed that the $\mathrm{MoH}$ and the CHAM Secretariat are involved in signing a memorandum of understanding at the policy level while SLAs are administered, through a decentralized structure, at the district level with the DHO taking charge of the implementation process. The $\mathrm{MoH}$ delegates SLA implementation to the relevant DHOs who are responsible for providing funds for SLA services and salary costs of health workers. The DHO procures essential drugs, equipment, medical supplies, transport for referrals and other resources.

The results further revealed that the government, through the $\mathrm{MoH}$, initiated the reform engaging $\mathrm{CHAM}$ to draw up terms of operation. The reform was reported 
to have started quickly without any prior analysis of the political, economic and social contexts or identification of the necessary supporting structures and systems required for its effective and efficient implementation. Furthermore, it was reported that there are no clear procedures, communication channels, monitoring and evaluation systems to guide and support the implementation of SLAs. Additionally, communication was reported to be often informal and sometimes infrequent. It was also reported through facility level interviews that $\mathrm{DHO}$ and CHAM facility management, other civil societies (local and international NGOs) and health care providers were not consulted on the initiated reform. They were also not oriented on their expected roles.

\section{Reasons for introducing SLAs between the government and CHAM in Malawi}

Semi-structured interviews with central level and facility level managers revealed that SLAs were required, through a common interest between the government and CHAM, to improve access, equity and quality of maternal and neonatal services with the ultimate aim of reducing maternal and neonatal mortality and morbidity; this was also supported by the Development Partners. It was further reported that the common motivation for the SLA partnerships focused on the need to increase coverage and affordability in order to improve health and universal access to health care by the poor. Such overlapping interests are an important help to effective contracting and lower the risk of a moral hazard.

\section{Payment mechanisms within SLAs}

Through central and facility level semi-structured interviews it was found that payment mechanisms within SLAs are agreed upon through a reimbursement system and billing is based on the cost of services. There were conflicting views on whether SLAs had guidelines for the billing prices of services; whilst the price list was reported to be available, no guidelines were provided alongside them. MoH, in consultation with the CHAM Secretariat, drew up the original price list that was communicated to DHOs and CHAM facilities. Billing for care provided was, as noted, based on the cost of services following these agreed billing prices and the stated guidelines. There was a universal agreement among facility level managers of frequently "delayed cash transfers from the [state] treasury to the DHO", with consequent delayed payments to CHAM facilities.

\section{Efficiency, effectiveness, equity and sustainability of SLAs in Malawi}

There was unanimous agreement among participants involved in central and facility level semi-structured interviews and FDGs that SLAs were "a good initiative" and that they had helped overcome geographical barriers, increase coverage of health care services, improve health and universal access to health care for the targeted poor, and improve equity of access to health care services. As one health care provider at a CHAM facility mentioned:

"SLAs are good, we are serving poor people. The number of hospital visits has increased. Because of the SLA, people are willing to come because the government is going to pay, so we are saving lives.... previously, we were having more maternal deaths because of the delay in coming to the hospital, they were afraid to come, but with this SLA, it is reducing the maternal deaths. So people are coming earlier to the hospital and even to the antenatal clinic the number of people visiting has increased".

(Health care provider).

Improvements in the quality of health services over time was also recognized as a success of SLAs by patients through exit interviews. Findings from the client exit survey showed that clients used the services more after SLA implementation because the services were generally free; "not having to pay" had a great impact on the local service users and most of them reported satisfaction with the quality of care. Although the quality of data from HMIS was poor, it was still possible to spot the increase in number of children and women utilizing health care services due to the introduction of SLA services. In the Mtengowanthenga health facility the number of paediatric visits increased by approximately $600 \%$ from 2008 to 2010 and the number of deliveries increased by approximately $400 \%$ from 2004 to 2010 (Figures 1 and 2).

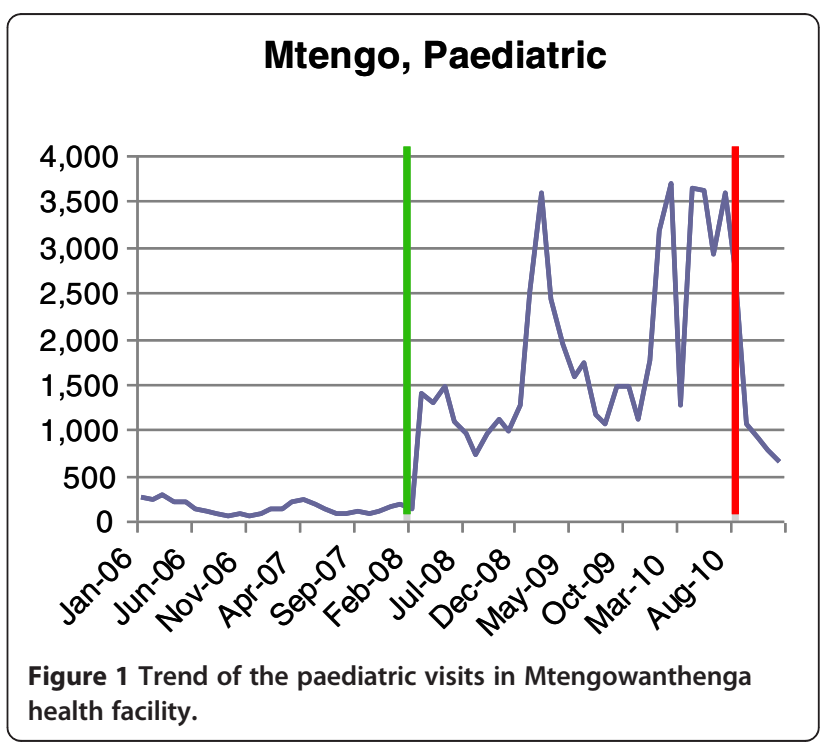




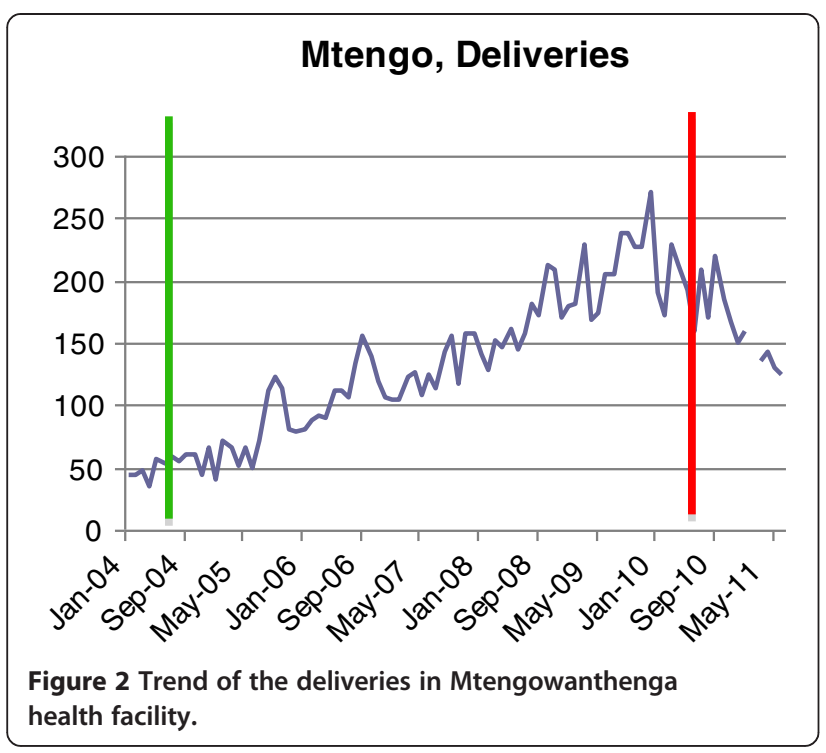

\section{Obstacles to SLA adoption and retention}

Through FGDs and semi-structured interviews with facility level managers, a lack of clear systems, guidelines, policies, procedures, and role clarification among stakeholders was reported to have negatively affected SLA performance. The hasty decision to implement SLAs was also reported as an obstacle to allowing adequate and effective preparation for SLA implementation and this overwhelmed the providers, institutions and stakeholders. Furthermore, a lack of transparency, dishonesty, failure to follow guidelines and overcharging of SLA services, were also reported to have a negative impact on the performance of SLAs. As one respondent was quoted as saying:

\begin{abstract}
"When DHO was doing data verification [at CHAM], we noticed that some clients did not appear in the HMIS register, and some clients were males. The statistics for SLA patients does not change despite variations in disease epidemiology. Every month the figures are the same. For example, from January to December the figures for Malaria cases are more or less the same despite that in some months the incidence of Malaria is higher compared to the other months". (Health care provider).
\end{abstract}

The facility level managers also reported that inadequate revenues to fund SLAs led to delayed cash transfers and payments and placed a strain on the relationship between CHAM facilities and the DHOs. Furthermore, it was reported that SLA billings do not include the cost of overhead costs (utilities), such as electricity, water, fuel and telephone bills; as such, CHAM depends on other charges such as consultation fees to cover up the overhead costs. The cost of overheads and consultation fees therefore emerged as a prohibiting factor to effective functioning of SLAs. As one respondent indicated:

"...this still remains a paying institution though [it] is under SLA...overhead costs are not included in the SLA bills. The government is not giving us fuel for vehicles and is not paying electricity for us, the government does not pay for our phones, maintenance of structures, now how do we sustain those activities if we only get drugs. You see now, though we are paid, we appreciate we are paid. We still need to maintain the institution. Apart from giving the medication to the patients, the other operations have to move". (CHAM facility manager).

Furthermore, the facility level managers reported that the lack of a validation system in CHAM's health records and documentation contributed to deterring efficiency, which was made worse by a lack of supervision, breeding further mistrust between DHOs and CHAM facilities. This led to the DHO accumulating debt. Subsequently SLAs were cancelled and in many cases this was reportedly due to non-payment of bills. Where facility HMIS data exist, they show that SLA cancellation resulted in reduced utilisation of services. In March 2008, an SLA was put in place to provide paediatric care in Mtengowanthenga health facility and, in September 2010, it was decommissioned. HMIS data showed that SLA enactment prompted a significant increase in the use of paediatric services (more than a 600\% increase) from 2008 and the use of the services dropped back to the original level following this decommissioning in 2010 (Figure 1). Similarly, the number of deliveries reported by HMIS, in the Mtengowanthenga facility steadily increased over the last seven years following SLA implementation for delivery service (2004) while they declined when the SLA was decommissioned in 2010 (Figure 2).

Rising costs of services were also identified to be an important factor. HMIS data for Mulanje Mission hospital, between 2006 and 2011, shows that there was an increase of $23 \%$ in the utilization of maternal services and this resulted in a $56 \%$ increase in costs for these services. Apart from the increase in utilisation of SLA services, other factors were also reported to have influenced this sharp rise in costs. Firstly, the increase was perversely triggered by the non-revision of the initial price list, drawn up five years previously. CHAM facilities reported to have raised their concerns with DHOs, but, with no tangible response, they felt compelled to revise prices unilaterally. According to the signed memorandum of understanding, the DHO cannot pay invoices beyond the prices originally agreed. This results in accumulation and non-payment of bills thereby acting as hindrance to provision of SLA forcing 
some facilities to suspend provision of SLA services. As one respondent mentioned:

"The bill is big and they are failing to pay. The number of referrals has been cut; we are no longer accepting referrals. If you go around you will see that there are no patients in the wards and it's because of that. We've told them that as long as they cannot accept to pay us using the bill we've used to buy drugs, then we cannot provide services, or else the hospital is going to collapse. We have told them that for the mean time they should not send patients. We are only receiving paediatric and maternity cases on humanitarian grounds, but we are even losing more money". (CHAM facility manager).

Secondly, the facility level managers reported that according to the SLA, CHAM facilities were to procure their drugs from the Central Medical Stores; however, when the stores could not supply them with drugs, CHAM facilities ended up buying from private drug suppliers whose prices are normally expensive compared to Central Medical Store charges. One facility level manager explained as follows:

"When we are signing the agreement we are supposed to be collecting drugs from the Central Medical Stores, now when you go there you find that drugs are not there, instead we go to private pharmacies to buy the drugs obviously at a higher price. When billing, we bill at those higher prices, even though when we were signing the agreement there was this price list which we were supposed to adhere to. When paying the bill, they only pay us based on the old prices which came in 2006, which are lower... For example if we buy drugs at K1,000.00, and when billing if they refer to the old price list you will find that it is 300 or 400 which is a loss now. When we ask them to pay us the difference...there is that misunderstanding and we struggle to get the answer". (CHAM facility manager).

A substantial increase in workload due to SLA introduction was also identified as a problem affecting the implementation of SLAs. The 'Front Line Providers' survey carried out with the employees demonstrated that they perceived that "their workload had increased as a result of the SLA": the average facility score was 4.3 out of maximum of 5 . The facility level managers reported that the workload was compromising quality of care because initially there were no additional resources for the anticipated number of clients. One health care provider said:

"With SLA the workload has increased....we have limited human resources... with the same number of staff we have to take care of twenty or hundred times of the patients. The workload is too much, the congestion is too much". (Health care provider).

A facility level semi-structured interview revealed that compared to the time when SLAs were introduced, there is now decreased enthusiasm pertaining to the SLAs because of delayed payments and review of contracts, as well as the presence of different unresolved misunderstandings between government and CHAM. One respondent shared his observation on this as follows:

"I think the time the SLA idea was being developed everyone was very happy, motivated and dedicated, because things started well and were just running smoothly. Now things are changing, I can say there is not much enthusiasm pertaining to the SLA's".

(Health care provider).

\section{Discussion}

There is strong consensus and shared interest between the government and CHAM regarding SLA and the extension of universalization. This was very helpful to the formulation of the contracts and their initial implementation. Further, it appears that free services provided by SLAs improved utilization. SLAs helped to overcome geographical and financial barriers to access antenatal, maternal and infant care thereby improving equity of access to health services.

However, the findings show that the performance and continuation of SLAs in Malawi is being affected by a lack of supporting processes and structures. The key concerns are non-revised prices, late payment of bills, a lack of transparency, poor communication within and among stakeholders including health care providers, civil societies and communities, inadequate human and material resources and lack of monitoring systems and general guidelines. The findings of this study reveal that SLAs were introduced quickly without any prior analysis of the political, economic and social contexts and without adequate supporting structures, such as clear policies to guide implementation and arbitration committees to resolve contractual difficulties. All this has created growing mistrust, moral hazard, and in some cases, the termination of SLAs. Such results confirm key findings from the international literature $[6,12,13]$.

This situation raises serious concerns about the effectiveness and sustainability of SLAs in Malawi without remedial action. The lack of documentation, unverified records, and inadequate monitoring gave the facilities the freedom to fluctuate their cost figures. In particular, billing prices for SLA services increased sharply to the extent that a bill from a single CHAM facility exceeded the total budget of the DHO. At first sight, the case 
studies suggest that contracting may prove more expensive than equivalent government provision [12]. Yet, on closer inspection, a complex picture emerges from the study and it is not easy to tell what an appropriate level of funding would be. Many CHAM facilities are in remote areas where unit costs will necessarily be higher. Where the government had committed to supplying drugs and this did not happen, CHAM facilities incurred higher costs as they sourced from private suppliers. In addition, postponed government payments may have produced deliberate cost escalation from NGO facilities to compensate for the delays. Further, the financial effect of the large increase in the volume of services in some cases does not seem to have been anticipated. More fundamental research is required to highlight better pricing tariffs for such contracts.

The signing of SLAs did not stop user fees (for maternal, neonatal and infant care) in some cases and some CHAM facilities continued to raise revenues from patients for services provided. It is important to highlight that individual perceptions of a public policy are essential for evaluating the success or failure of any program or system [14]. Consequently, the case studies show that contracting can be effective in improving the quality and coverage of care [15], as well as improving the health system's overall performance, when correct supporting measures are taken. Due to many challenges with the availability and quality of data, the study failed to carry out detailed individual facility analyses of the efficiency and equity of purchasing for SLAs. The study did not manage to collect continuous data from all case study sites. Data was not available due to poor documentation and record keeping.

On reflection, there were clearly overlapping objectives across the parties in the provision of good free care but this was not the whole picture. Breaches of trust occurred on both sides in terms of incidents of not supplying finances or medicines at the appropriate time on the government side and not removing user fees and purchasing costly medicines for care in some CHAM facilities. The matter of resource availability and sustainability may have proved more important to each party than delivering care in particular cases. Further, the early trust brokered by common objectives seemed to disintegrate over time as parties learned of each other's actions and adjusted their own responses. Such experiences of contracting reinforce many findings in the literature. Good information flow between parties was not established or enabled at the outset, exacerbating information imbalances and moral hazard. An effective evaluation system for verifying CHAM's records was absent. Such factors may have made it easier for parties to evade their responsibilities. Further transaction costs were higher than necessary because of a lack of formal support to the SLAs. The Malawian experience highlights the importance of providing good support to help make contracting more effective and of facilitating required information and resource flows. These results can provide guidance, lessons and recommendations to policy makers (MoH, CHAM Secretariat) in replicating and strengthening the implementation of SLAs in the roll-out of universalization policy and to other contexts attempting similar initiatives.

\section{Recommendations}

There is a need to review, develop and implement comprehensive guidelines with clear time lines, targets, roles and responsibilities of players at different levels, such as DHOs, CHAM Secretariat, CHAM facilities and communities. It is important to develop a contracting framework and strengthen the data management system with indicators for measuring and monitoring performance. Furthermore, there is a need to establish a simple but robust financial reporting mechanism to keep costs updated (including maintenance costs), and to develop clear billing and payment mechanisms. A trusting relationship between the contracting parties is essential for effective implementation of SLAs. Policy makers should make a deliberate effort to involve civil society at all levels and establish and strengthen (procedures) processes with regard to resource allocation, communication, record keeping and reporting. All stakeholders involved in the SLA implementation should be consulted on what is possible and what is not, in terms of the feasibility of the SLA, predictable obstacles and barriers, as well as strengths.

\section{Abbreviations}

CHAM: Christian Health Association of Malawi; DHO: District health office; EHP: Essential health package; FGD: Focus group discussion; HMIS: Health management information system; MoH: Ministry of Health; SLA: Service level agreements.

\section{Competing interests}

The authors of this report had no significant financial, professional or personal interests that might have influenced the performance or presentation of the work described in this report.

\section{Authors' contributions}

The work presented here was carried out in collaboration between and among all authors. The Principal Investigator, Associate Professor Maureen Leah Chirwa and the Co-Principal Investigator Associate Professor Steve Thomas defined the research theme and its protocol. The PI facilitated the fieldwork, data management and analysis. Both the PI and Co-PI worked together on the report with the Research Fellows: Isabel Kazanga and Giulia Faedo. All authors have contributed to, seen and approved the contents of the report.

\section{Acknowledgements}

We acknowledge that this study is financially and technically supported by the Alliance for Health Policy and Systems Research, WHO. We also wish to acknowledge the inputs of the Health Systems Financing Department, WHO and the late Guy Carrin, in particular. We are also grateful to the Alliance for the capacity building of the $\mathrm{PI}$ and $\mathrm{Co}-\mathrm{PI}$ during the proposal development workshop. We would also like to thank Malawi MoH and CHAM for

facilitating the research team to collect data in their sampled health facilities 
and district hospitals. We also would like to thank the SLA National Steering Committee for overall direction and support with literature review documents and selection of case study areas. Lastly, we are greatly indebted to our study participants, for without them our study would not have been possible.

\section{Author details}

${ }^{1}$ Community Health Department, College of Medicine, Chichiri, Blantyre 3, Malawi. ${ }^{2}$ Community Health Department, University of Malawi, College of Medicine, P/B 360, Chichiri, Blantyre 3, Malawi. ${ }^{3}$ Centre for Health Policy and Management, Centre for Global Health at Trinity College Dublin, Foster Place, Dublin 2, Ireland

Received: 20 January 2013 Accepted: 29 July 2013

Published: 19 August 2013

\section{References}

1. Statistical Office (NSO) [Malawi] and ICF Macro: Malawi Demographic and Health Survey 2010: Preliminary Report. Calverton, Maryland: NSO and ORC Macro; 2011.

2. Ministry of Health: Malawi Health Sector Strategic Plan 2011-2016. Malawi: Lilongwe; 2011.

3. Mueller DH, Lungu D, Acharya A, Palmer N: Constraints to implementing the Essential Health Package in Malawi. PLoS One 2011, 6(6):e20741.

4. Zere E, Moeti M, Kirigia J, Mwase T, Kataika E: Equity in health and healthcare in Malawi: analysis of trends. BMC Publ Health 2007, 2007:7-78.

5. Levisohn B, Harding A: Buying results? Contracting for health service delivery in developing countries. Lancet 2005, 366:676-680.

6. Palmer N, Mills A: Contracting out health service provision in resource- and information-poor settings. In Elgar Companion to Health Economics. Edited by Jones AM. Cheltenham: Edward Elgar; 2006:250-260.

7. Palmer N, Strong L, Wali A, Sondorp E: Contracting out health services in fragile states. Br Med J 2006, 332(7543):718-721.

8. Roberts MR: The Role of Dynamic Renegotiation and Asymmetric Information in Financial Contracting (August 27, 2012). 7th Annual Conference on Empirical Legal Studies Paper; 2012. [http://papers.ssrn.com/sol3/papers. cfm?abstract_id=1732364] Accessed 22 ${ }^{\text {nd }}$ April 2013.

9. Alliance for Health Policy and Systems Research and World Health Organization: Identifying Priority Research Questions, Theme 3: Non-State Sector Issues in Low and Middle-Income Countries; 2008 [http://www.who.int/ alliancehpsr/researchsynthesis/Alliance_HPSR_NonStateSector_TwoPager.pdf].

10. Walt G, Shiffman J, Schneider H, Murray SF, Brugha R, Gilson L: Doing' health policy analysis: methodological and conceptual reflections asnd challenges. Health Policy Plan 2008, 23:308-317.

11. Yin RK: Case Study Research. Design and Methods. Applied Social Research Methods Series. Thousand Oaks: Sage Publications; 2008.

12. Boulenger D, Keugoung B, Criel B: Contracting between Faith-based and Public Health Sector in Sub-Saharan Africa: The Case of Cameroon, Tanzania, Chad and Uganda. Antwerp: Institute of Tropical Medicine; 2009.

13. Bastias G, Rada G: Does Contracting Out Services Improve Access to Care in Low and Middle Income Countries? A SUPPORT Summary of a Systematic Review 2008; 2008 [http://www.support-collaboration.org/summaries.htm]

14. Murray A: Public Perceptions Versus Good Public Policy (Commentary); 2003 [http://www.highbeam.com/doc/1G1-81223393.html]

15. National Statistical Office: Population Projections for Malawi, 2012. Malawi: Zomba; 2012.

doi:10.1186/1478-4505-11-27

Cite this article as: Chirwa et al:: Promoting universal financial protection: contracting faith-based health facilities to expand access lessons learned from Malawi. Health Research Policy and Systems 2013 11:27.

\section{Submit your next manuscript to BioMed Central and take full advantage of:}

- Convenient online submission

- Thorough peer review

- No space constraints or color figure charges

- Immediate publication on acceptance

- Inclusion in PubMed, CAS, Scopus and Google Scholar

- Research which is freely available for redistribution

Submit your manuscript at www.biomedcentral.com/submit
C Biomed Central 